\title{
Nanomaterials and their applications
}

\author{
Fehim Findik ${ }^{1,2}$ \\ ${ }^{1}$ Metallurgy and Materials Engineering Department, Faculty of Technology, Sakarya Applied Sciences \\ University, Sakarya, Turkey \\ ${ }^{2}$ BIOENAMS R\&D Group, Sakarya University, Sakarya, Turkey
}

\begin{abstract}
The word nano takes its meaning from the Greek word "nanos" which means dwarf. Nanoparticles are materials ranging in size between 1 and 100 nanometers. A nanometer is one billionth of a meter. Nanotechnology is an interdisciplinary field that researches the physical, chemical and biological structures of materials with dimensions of one billionth and deals with their usage areas. Nanomaterials are the cornerstones of nanotechnology and they possess unique optical, magnetic and electrical properties in this dimension. What makes nanotechnology so interesting is that materials behave differently from the macro world in this dimension. Power / weight ratio, conductivity, optical and magnetic properties change significantly as we move from macro dimension to nanosize. In this study, after giving general information about nanomaterials, nanotechnology processes, imaging techniques of nanomaterials, carbon nanotubes, nanocomposites, nanotechnology in nature and various nanotechnology applications have been investigated.
\end{abstract}

\begin{tabular}{l} 
Keywords: Nanomaterials, nanotechnology, carbon nanotubes, nanocomposites, applications \\
\hline Corresponding Author: \\
Fehim Findik, \\
Metallurgy and Materials Engineering Department, Faculty of Technology, Sakarya Applied Sciences \\
University, Sakarya, Turkey \\
BIOENAMS R\&D Group, Sakarya University, Sakarya, Turkey \\
E-mail: findik@ sakarya.edu.tr
\end{tabular}

\section{Introduction}

New technologies that work, apply or operate dimensionally at "nanometer" levels are collectively called "Nanotechnology". Nanotechnology is controlling and engineering of scientific results resulting from the emergence of new properties and brand new properties of matter at nanoscale (one billionth of a meter). Nanoscale is a material size approx. $1 \mathrm{~nm}-100 \mathrm{~nm}$ (Figure 1). Nanostructure can be 1D, 2D or 3D dimensions at nanoscale. Nanoparticle is a nanoscale matter / material in 3 dimensions and nanoscale. Nanocomposite is at least one component in a composite structure consisting of different materials or chemicals at the nanoscale (one nanoparticle and another nanostructure).

At the nano level, the physical, chemical and biological properties of matter differ fundamentally and significantly. There are radical changes in the properties of individual atoms, molecules and mass. In accordance with the quantum theory, nanometer-sized matter changes from "particle-like" to "wave-like" behavior.

We come across examples of "nanotechnological" applications within thousands of years of technological know-how. In this regard, another discussion centers around the issue that "nanotechnological" scientific and technological innovations are not so new. A striking example of this is the famous Lycurgus Cup. There are bronze figures depicting King Lycurgus added in later periods in the glass cup from the Roman period of the 4th century AD. The most important feature of the mug is its color that changes with the light.

When many properties of substances / materials such as physical, chemical, mechanical, thermal and magnetic are examined, it is known that they vary greatly in nanosize $(1-100 \mathrm{~nm})$. Among the reasons for this, especially the ratio of surface area to unit volume (YA / V) and / or unit weight emerges as an important factor. As the YA

(C) The Author 2021. This work is licensed under a Creative Commons Attribution License (https://creativecommons.org/licenses/by/4.0/) that allows others to share and adapt the material for any purpose (even commercially), in any medium with an acknowledgement of the work's authorship and initial publication in this journal. 
I V ratio increases, most atoms in an atomic lattice are located at or near the surface. This means that most atoms are bound together by weaker bonds, due to the atoms that are not open to the outside [1].

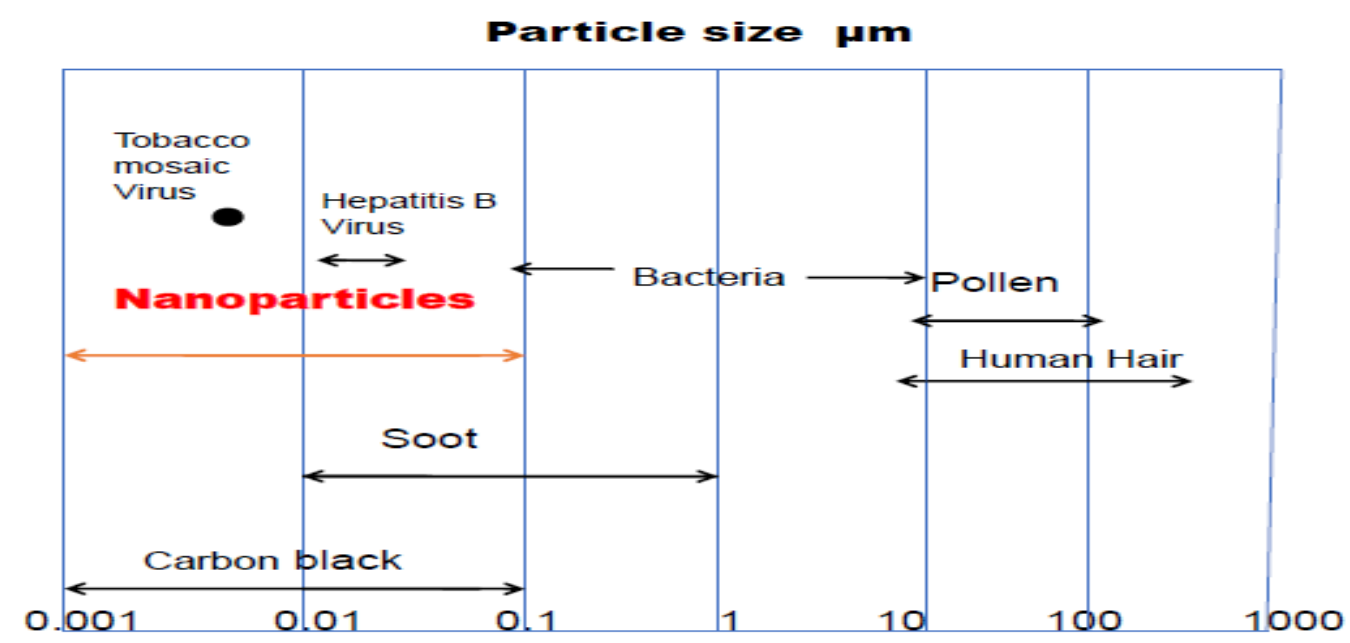

Figure 1. Particle sizes and the scales for various objects

About the classification, nanomaterials have extremely small size which having at least one dimension $100 \mathrm{~nm}$ or less. Nanomaterials can be Nanoscale in one dimension, two dimensions, or three dimensions. They can exist in single, fused, aggregated or agglomerated forms with spherical, tubular, and irregular shapes [2].

Common types of Nanomaterials include nanotubes, quantum dots and fullerenes. Nanomaterials have applications in the field of Nanotechnology, and displays different physical chemical characteristics from normal chemicals (i.e., Silver Nano, Carbon Nanotube, Fullerene, Photo Catalyst, Carbon Nano, and Silica) [3].

On the basis of dimension, nanomaterials are classified as follows:

- Zero dimensional nanostructures (e.g. Spheres and Clusters etc.)

- One dimensional nanostructure (e.g. Film. Coatings, Multilayer's etc.)

- Two dimensional nanostructures (e.g. Tubes, Fibers, Wires, Platelets etc.)

- Three dimensional nanostructures (e.g. Particles, Quantum Dots, Hollow Spheres etc.)

Nanomaterials are materials which are characterized by an ultra-fine grain size $(<50 \mathrm{~nm})$ or by a dimensionality limited to $50 \mathrm{~nm}$. Nanomaterials can be created with various modulation dimensionalities. Zero (atomic clusters, filaments and cluster assemblies), one (multilayers), two (ultrafine-grained over layers or buried layers), and three (Nanophase materials consisting of equiaxed nanometer sized grains) as shown in the above Figure 2.

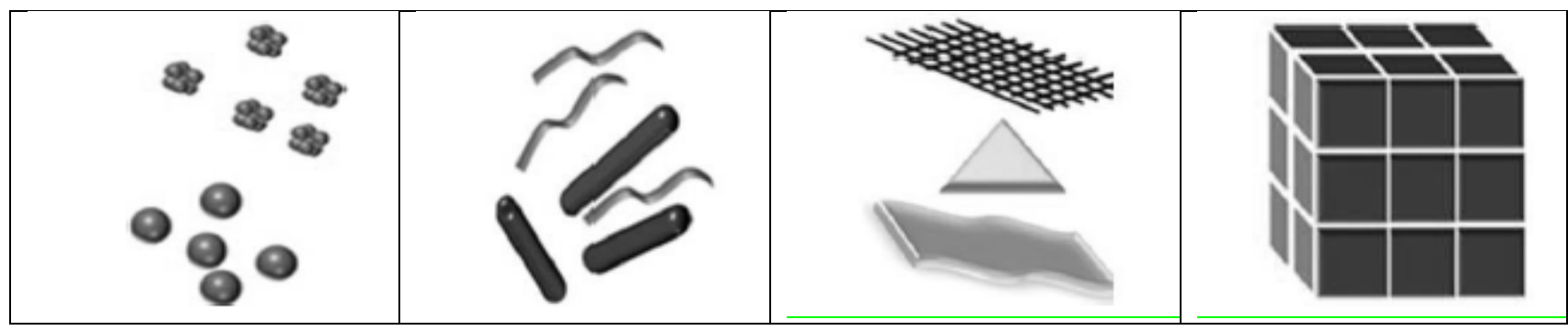

Figure 2. Types of nanomaterials; a) 0D Spheres and Clusters, b) 1D Nanofibers, Wires, and Rods, c) 2D Films, Plates, and Networks, d) 3D Nanomaterials [3]

On the basis of phase composition, nanomaterials are classified as follows:

- Single Phase Solids (e.g. Crystalline, Amorphous Particles and Layers etc.)

- Multi-Phase Solids (e.g. Matrix Composites, Coated Particles etc.)

- Multi-Phase System (e.g. Colloids, Aerogels, Ferro fluids etc.) 
On the basis of manufacturing process, nanomaterials are classified as follows:

- Gas Phase Reaction (e.g. Flame Synthesis, Condensation, CVD etc.)

- Liquid Phase Reaction (e.g. Sol-Gel, Precipitation, Hydrothermal Processing etc.)

- Mechanical Procedures (e.g. Ball Milling, Plastic Deformation etc.)

About the properties of nanomaterials, the chemical and physical properties of Nanomaterials (such as optical absorption and fluorescence, melting point, catalytic activity, magnetism, electric and thermal conductivity, etc.) are typically differ significantly from the corresponding coarser bulk material. A wide range of material properties can be adjusted by structuring at the Nanoscale.

Table 1. Adjustable Properties of Nanomaterials Properties Examples [3]

\begin{tabular}{|l|l|}
\hline Properties & \multicolumn{1}{|c|}{ Examples } \\
\hline Catalytic & Better catalytic efficiency through higher surface-to volume ratio. \\
\hline Electrical & $\begin{array}{l}\text { Increased electrical conductivity in ceramics and magnetic Nano composites, increased electric } \\
\text { resistance in metals. }\end{array}$ \\
\hline Magnetic & Increased magnetic coactivity up to a critical grain size, super paramagnetic behavior. \\
\hline Mechanical & Improved hardness and toughness of metals and alloys, ductility and super plasticity of ceramic. \\
\hline Optical & $\begin{array}{l}\text { Spectral shift of optical absorption and fluorescence properties, increased quantum efficiency of } \\
\text { semiconductor crystals. }\end{array}$ \\
\hline Sterical & Increased selectivity, hollow spheres for specific drug transportation and controlled release. \\
\hline Biological & Increased permeability through biological barriers, improved biocompatibility. \\
\hline
\end{tabular}

In this study, the nanomaterials and nanotechnology are briefly defined, short development history is given, images for the nanomaterials are briefly explained, carbon nanotubes and nanocomposites are explained, and finally application areas for the natural and industrial applications are briefly explained.

\section{Nanotechnology processes and manufacturing technologies}

Nanoscience involves studies on well-defined nanostructures or nanomaterials by combining materials at nanoscale between about 1-100 $\mathrm{nm}$ and the basic building blocks at the two- and three-dimensional molecular level. Generally, nanoparticles are considered under two main groups. These can be summarized as follows: Carbon-based materials such as carbon nanotubes or fullerenes Inorganic nanoparticles: metal oxides (such as zinc oxide, iron oxide, titanium dioxide and cerium oxide), metals (gold, silver and iron) and quantum dots "quantum dots" (cadmium sulfide and cadmium selenide) [4].

Gas phase processes (aerosol processes) are the most important processes used on an industrial scale for obtaining nanomaterials in powder or film form. Nanoparticles are obtained from the gas phase formed by chemical or chemical means. The nanoparticles formed in the first stage are formed by homogeneous nucleation mechanism in liquid or solid state.

Depending on the process, for further magnification of the particles, the following stages of are applied: transition from gas to dense liquid by condensation; chemical reaction (s), on particle surfaces; condensation, adhesion of two or more parts (condensation); coalescence and fusion of particles (coagulation).

In liquid phase processes, wet-chemical synthesis of nanomaterials occurs at lower temperatures than gas phase. The most important liquid phase processes in nanomaterial production are: Precipitation, Sol gel processes, and Hydrothermal processes [5].

Photolithography techniques, traditionally used since the late 1950s, were used in such macro / micro circuit designs and constituted the main manufacturing process of the semiconductor industry. The patterns / templates are masked with films made of chrome on a quartz layer. Afterwards, it is exposed to UV rays as a photoresist layer as a light-sensitive material. Generally, the photoresist material in question is a polymeric material, designed to vary its solubility with a treatment solution under light. 
In order to better understand the "self-assembly" technique, if we start directly from the terminology, "self" means its own dynamics without outside help / support. "Assembly" means assembling or building / building. As can be understood from these terms, starting from the basic building blocks, structures that come together by themselves are the subject of discussion.

\section{Imaging in nanotechnology}

Microscopy techniques are techniques that give images of structures or details too small to be seen with the human eye. In order to be able to examine and investigate any object, it is of great importance to display the relevant structural elements in as much detail as possible with powerful microscopy techniques. Therefore, powerful electron microscope technologies continue to be developed with advanced technological equipment.

\section{TEM}

In Transmission Electron Microscopes (TEMs), the electron beams produced in the electron source are passed through an extremely fine sample prepared by special processes. During this transmission, the image is formed as a result of the interactions between the electron beams and the sample, it is enlarged and focused and reflected on a screen. As a screen, there is projection on a fluorescent screen or CCD camera. Extremely high magnifications are possible with TEM techniques. Thus, it is possible to visualize layers of atoms. It was stated that samples were prepared with extremely laborious, difficult and time consuming processes in TEM technique applications. Samples that need to be prepared extremely thin for electron beam transmission are generally 3 $\mathrm{mm}$ in diameter and less than $80 \mu \mathrm{m}$ in thickness.

Modern TEM systems (Figure 3), an example of which is shown in the figure, are quite expensive, difficult to operate and high cost. It has highly sensitive electronic and mechanical devices that require constant maintenance and review. Components of a TEM system can be listed as follows: Electron source; high voltage applied main source from which electrons are generated; Sample; Image rendering: image plane or back focal plane; Projection (magnification) of the image; - Viewing and recording on the image.

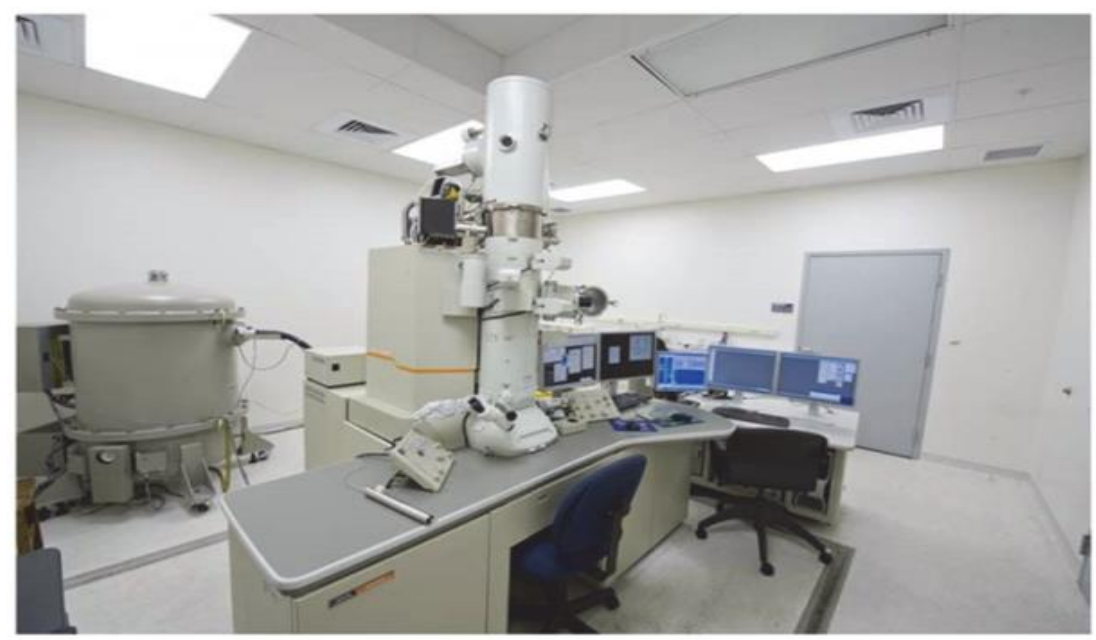

Figure 3. Image of a modern TEM microscope

\section{AFM}

Atomic Force Microscopy (AFM) technique reveals the atomic or nanoscale surface topography of a substance. With the AFM technique, a three-dimensional profile of a nanoscale surface can be created. It is a very powerful technique especially used in surface-based research of nanotechnologies. Atomic Force Microscopy (AFM) traces the topography of samples with extremely high - up to atomic - resolution by recording the interaction forces between the surface and a sharp tip mounted on a cantilever. AFM provides spatial information parallel and perpendicular to the surface. In addition to topographic high-resolution information, local material 
properties such as adhesion and stiffness can be investigated by analyzing tip-sample interaction forces (Figure 4).

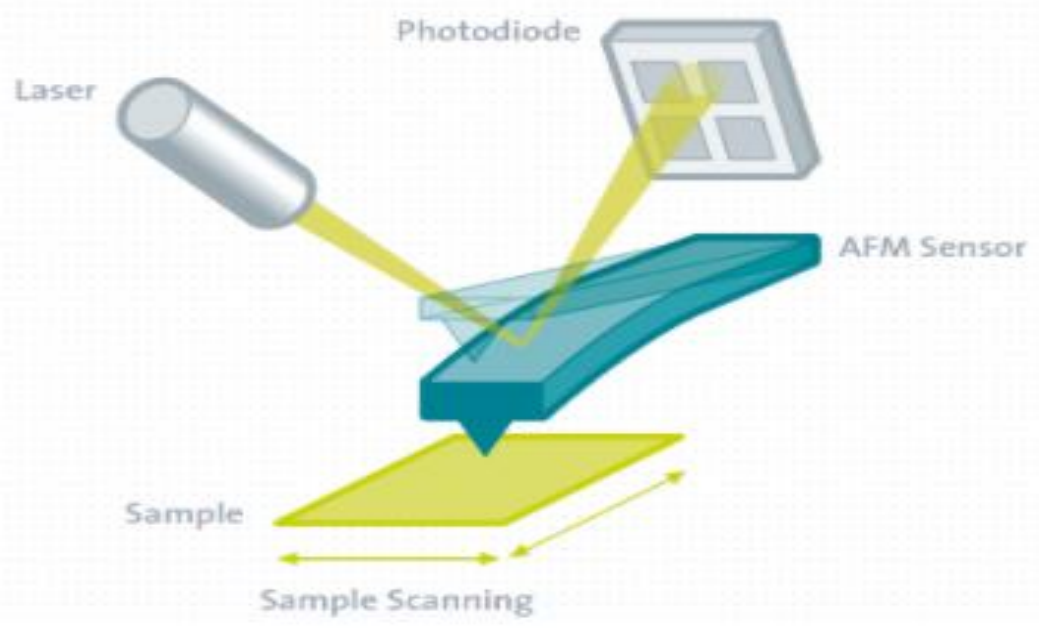

Figure 4. Atomic force microscope

\section{STM}

Scanning Tunneling Microscope (STM) (Figure 5) is one of the most important techniques for imaging samples at nanoscale. It also has the abilities to manipulate individual atoms as well as view it. It scans over the surface with a small voltage through a probe made of tungsten (the tip is 1-2 atoms thin). With the current on the probe tip, it detects any kind of indentation on the surface and scans the surface. With the current change, the surface topography in proportional relationship with it is displayed on the screen.

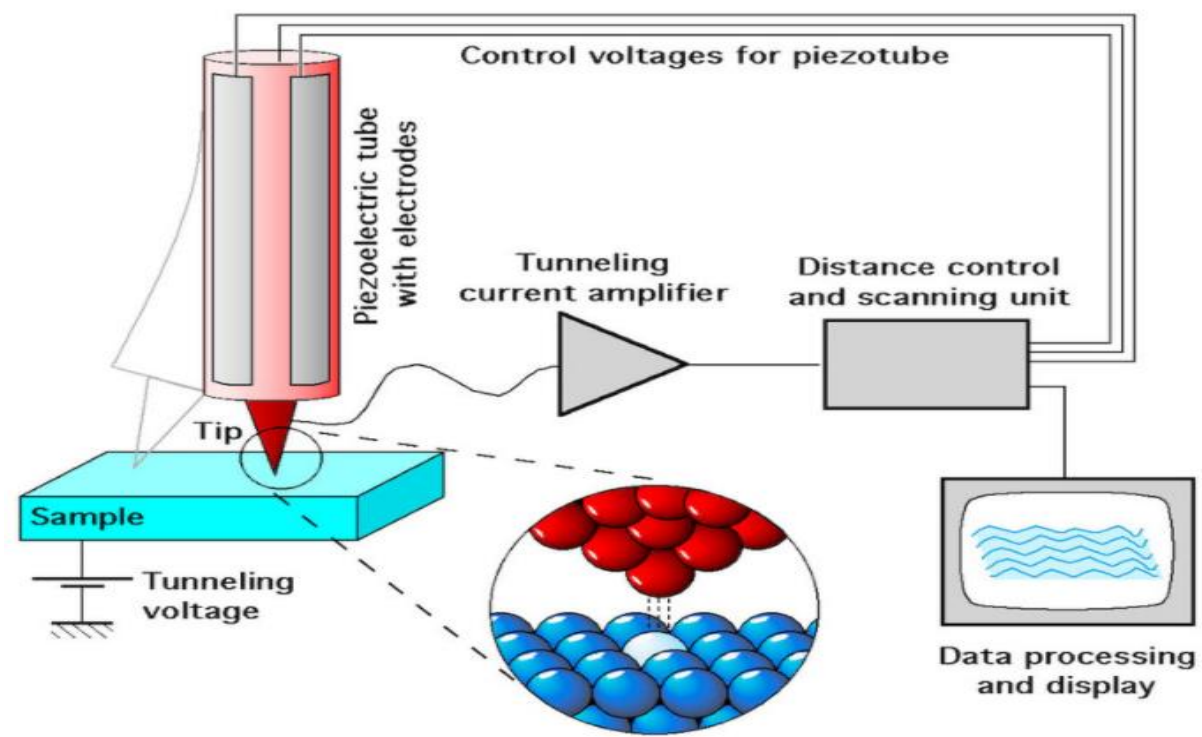

Figure 5. A modern scanning tunneling microscope (STM)

\section{Carbon nanotubes}

Carbon Nano Tubes (CNT) are cylindrical nanostructured carbon allotropes (Figure 6). It is formed by the bending of graphite plates (hexagonal form of carbon) into a cylindrical shape. The length: diameter ratio reaches unprecedented levels such as $=132000000 / 1$. This is a feature that cannot be found in any other substance or material. CNTs have very different properties and qualities: their electronic, optical, mechanical, thermal and other properties differ significantly from other materials. They can also be seen as elongated carbon "cylinders"; their diameter is less than $2.5 \mathrm{~nm}$, but their length can be of macroscopic size. It was first discovered and published by S. Lijima in Japan in 1991 [3]. 


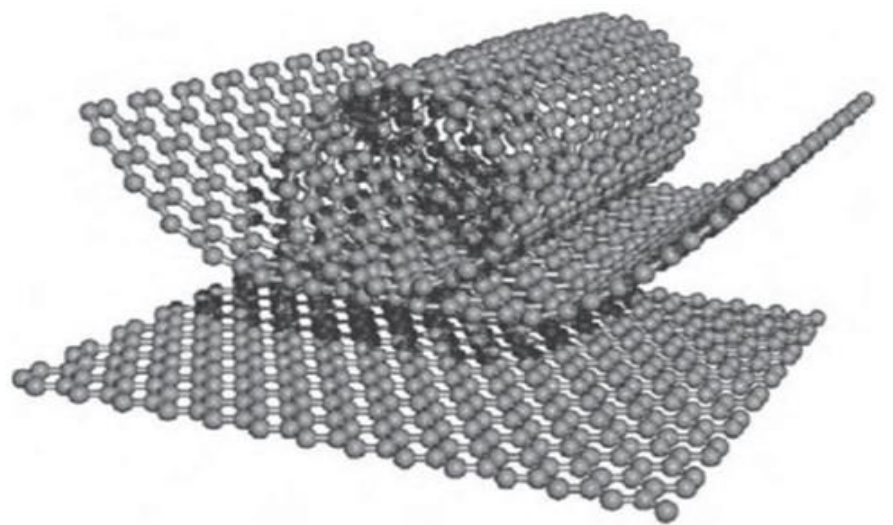

Figure 6 . A carbon nanotube is formed by crimping a graphitic insert into a cylindrical tube

Grafen

Graphene is the thinnest material (Figure 7). The carbon hexagon is tightly packed and has a single-atom thickness in a honeycomb-like planar layer of $\mathrm{sp}^{2}$ bonded atoms. Based on this, graphene was the first truly disclosed 2-dimensional crystalline material with extremely few errors.

The atomic structures of nanotubes can be defined by the chiral vector $\mathrm{C}_{\mathrm{h}}$ and chiral angle 0 created on the basis of tube "chirality" or "helicty". The most important universal known example of chirality is human hands. The left hand is an exactly "overlapping" mirror-symmetry image of the right hand. No matter what situation the hands are directed, both of them cannot exactly overlap and complete "overlap" cannot be achieved. This is similar to the difficulty encountered in wearing the left glove on the right hand. In terms of atomic configuration, a chiral molecule means a molecule that does not coincide in mirror symmetry, and generally an asymmetric carbon atom causes chirality.

Carbon-carbon bonds are the strongest in nature. Therefore, the carbon nanotube structure is a "perfect" arrangement of these strong bonds. It reaches extraordinarily high values of Young's Modulus (elastic constant). Carbon nanotubes stand out with their «inert» property, extremely high surface area, high tensile strength, low density, high thermal conductivity and flexible electronic behavior. They can also have high electron conductivity.

Among the extraordinary properties of carbon nanotubes are their very high electrical conductivity, thermal conductivity and mechanical properties. Apart from this, KNTs are the best electron field scattering materials. The extremely high level of length / diameter ratio plays a role in this. It is also important that it is well known as a pure carbon polymer and is suitable for many adaptations with its extremely rich chemistry.

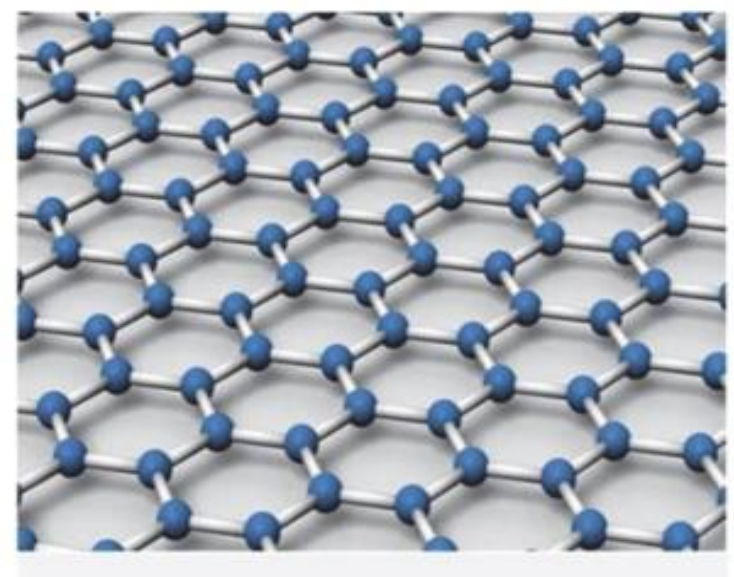

a)

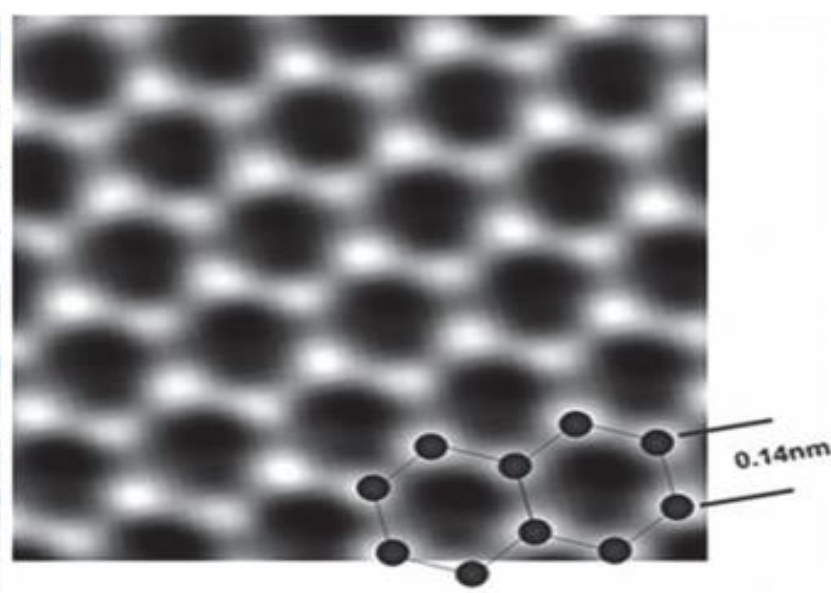

b)

Figure 7. a) Graphene representation of the graphene structure, b) High resolution TEM image of graphene [1] 


\section{Nanocomposites}

MMC

Metallic matrix (MMC) is relatively soft and flexible, as is generally seen in composites; On the other hand, reinforcement materials must have high strength and hardness. Since charges are transferred from the matrix phase to the reinforcement in the use of the composite, it is preferred that the bonds between the matrixreinforcement are as strong as possible and that there is some mutual solubility. In MMCs, two types of material for reinforcement are used in the form of particles, these are known particle reinforced composite structure and alloys reinforced with particle dispersion. In addition, $\mathrm{MMC}$ is produced with reinforcement phases in the form of long fibers.

\section{$\mathrm{CMC}$}

The ceramic matrix (CMC) phase is relatively hard and brittle, and the reinforcement components are capable of stopping crack propagation with high tensile strength. It is desirable that the reinforcement phase be capable of being removed from the matrix as the crack progresses; Therefore, interface bonds between matrix and reinforcement phases in CMCs should be weak.

PMC

The polymer matrix (PMC) should be relatively soft and flexible, while the reinforcement phase should have high strength and hardness. Since the loads are transferred from the matrix phase to the reinforcement in the use of the composite, certain interfacial operations are performed to ensure that the bonds between the matrixreinforcement are as strong as possible.

\section{Nanocomposites}

Nanocomposites are composite materials that have two or more components and at least one of the components is the size of the nanometer scale. Reinforcement phase of nanoscale materials in Polymer Matrix Nanocomposites or Polymer Nanocomposites (PNK), polymers (can be thermoplastics, thermosets or elastomers) are composite structures obtained by applying them. As nano-particle and nanofiber, the "l / h" aspect ratio should be greater than 300 .

Water vapor permeability is significantly reduced with nanocomposite coatings. In addition, a transparent coating is possible due to the clay particles homogeneously distributed in the polymeric matrix. In inorganicorganic composites based on organo-alkoxysilanes and other alkoxides, hard and high scratch resistant coatings are possible, especially on eyeglasses and lenses. With the addition of nanoparticles together with epoxy silanes, inorganic and organic cross bonds have been formed and a significant improvement has been achieved in abrasion resistance without changing transparency. At the same time, coatings with low surface energy can be made with nanocomposites.

\section{Nanotechnologies in nature}

\section{Nilufer (lotus) leaf effect}

The lotus grows in small lakes and ponds, ponds and puddles, and the surfaces of its leaves are always bright, clean and smooth, as if untouched by polluted water. It symbolizes pure cleanliness, as there is no bacteria and pathogen formation on the lotus leaves, which do not usually accumulate water on them. The water drops act like a drop of "mercury" on the lotus leaf and slide slowly over the surface, thus sweeping away any dirt and dust accumulated on the surface, leaving a clean surface behind (Figure 8). 


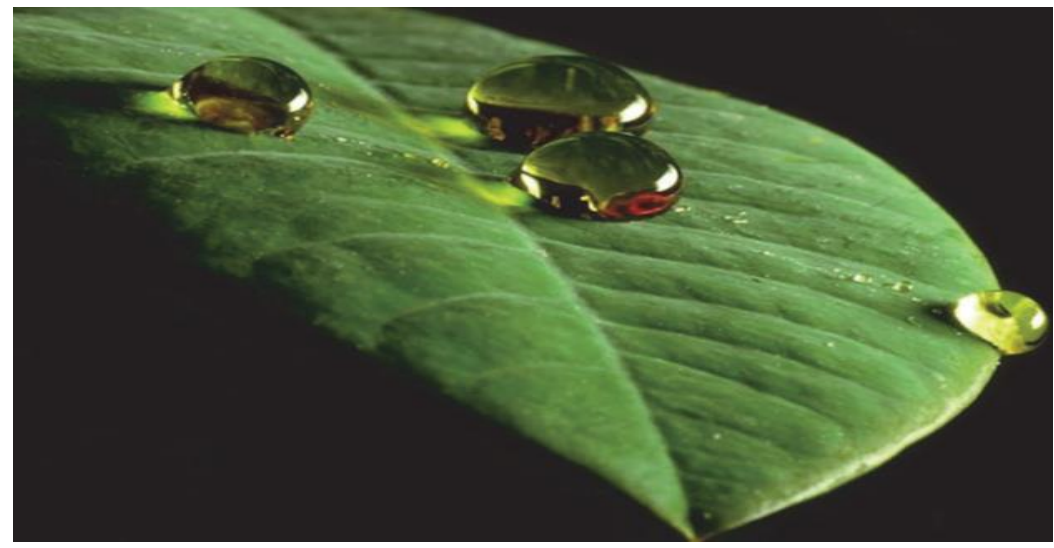

Figure 8. The view of lotus leaf

Based on the concept of the lotus effect, nanotechnology uses examples for industrial and everyday life (Figure 9):

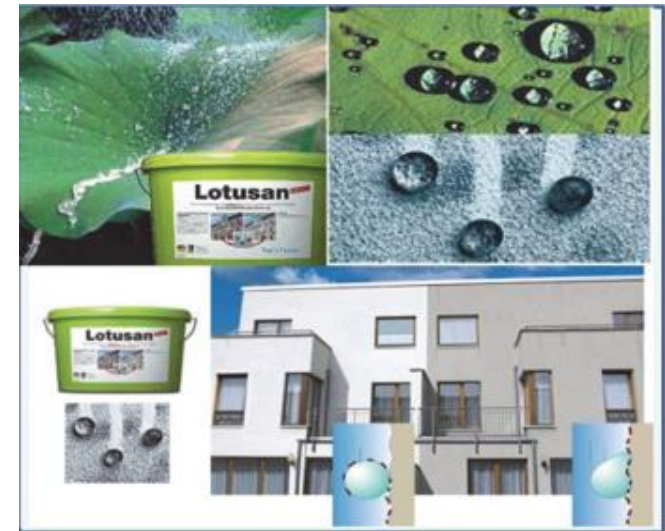

a)

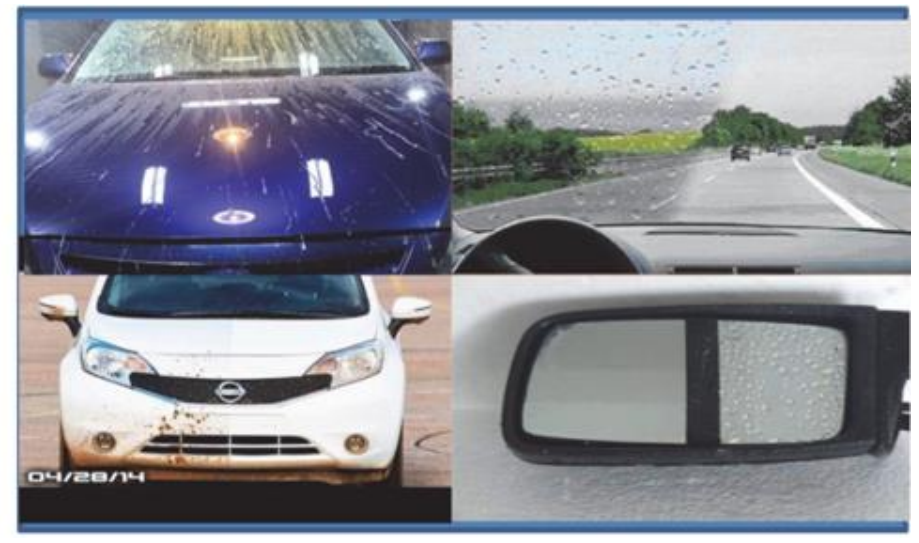

b)

Figure 9. Lotus effect applications in two sectors; a) dye sector, b) automobile industry [1]

\section{Gecko effect}

We know that many nanostructured formations exist in nature. As a result of billions of years, we are able to examine in detail the most developed form and structure examples that are most suitable for living conditions with modern electron microscopy techniques. One of these is the ability of lizards, known as the "Gecko Effect", to move rapidly in all conditions (humid, dusty, rainy, and other) vertically and inverted on any surface, defying gravity (Figure 10).

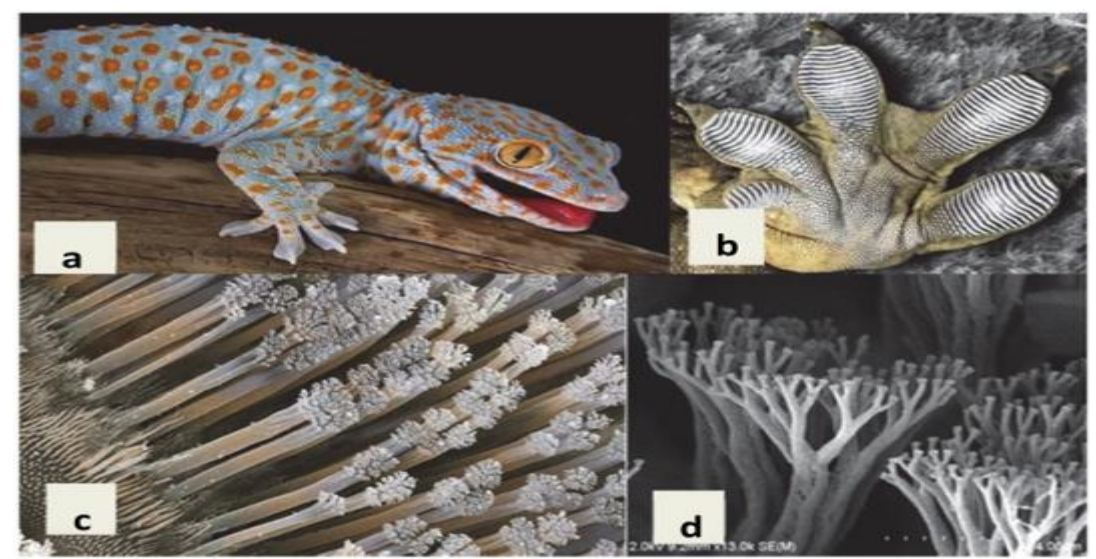

Figure 10. a) Gecko, b) bottom of the foot, c) hairy area at the bottom of the foot, d) SEM image of the hairs on the lower part of the gecko foot [1] 


\section{Nanotechnology applications}

Nanomaterials can be used in many different places (Figure 11).

-Automotive

Nano-structured materials, one of the most important areas of nanotechnology, are of great importance for the automotive industry. First of all, nanomaterials will play a critical role in efforts to reduce vehicle weight, higher strength and more flexible structures, as well as vehicle safety and security.

Steel is the most important structural material used in vehicle bodies in the automotive industry. The use of high-strength steels in vehicle bodies is being studied. However, in the cold forming of the steel, there are deviations in the dimensioning of the vehicle body. This problem has been tried to be overcome by hot forming. However, there is a problem of scale formation (rust layer caused by oxidation) on the surface due to the high temperature. Here, this can be remedied by nanotechnology and multifunctional coating on the surface. Bonded and bonded nano-sized glassy and plastic-like materials together with aluminum form a strong layer on the surface.

With nanotechnology, scratch-resistant, dirt-free and self-repairing vehicle paints can be applied on the exterior of the vehicle. This is done with nanoparticle flexible, fast-adhering, corrosion-resistant and antimicrobial layers in the nanocoating technique. Among the techniques given in the other page, anti-fogging, antifungal and antireflective coatings are also becoming widespread thanks to nanotechnologies.

With the rapid development of nanotechnologies, they will be used more intensively on the cars of the future within the framework of lightness, smart components and adaptable structures [6].

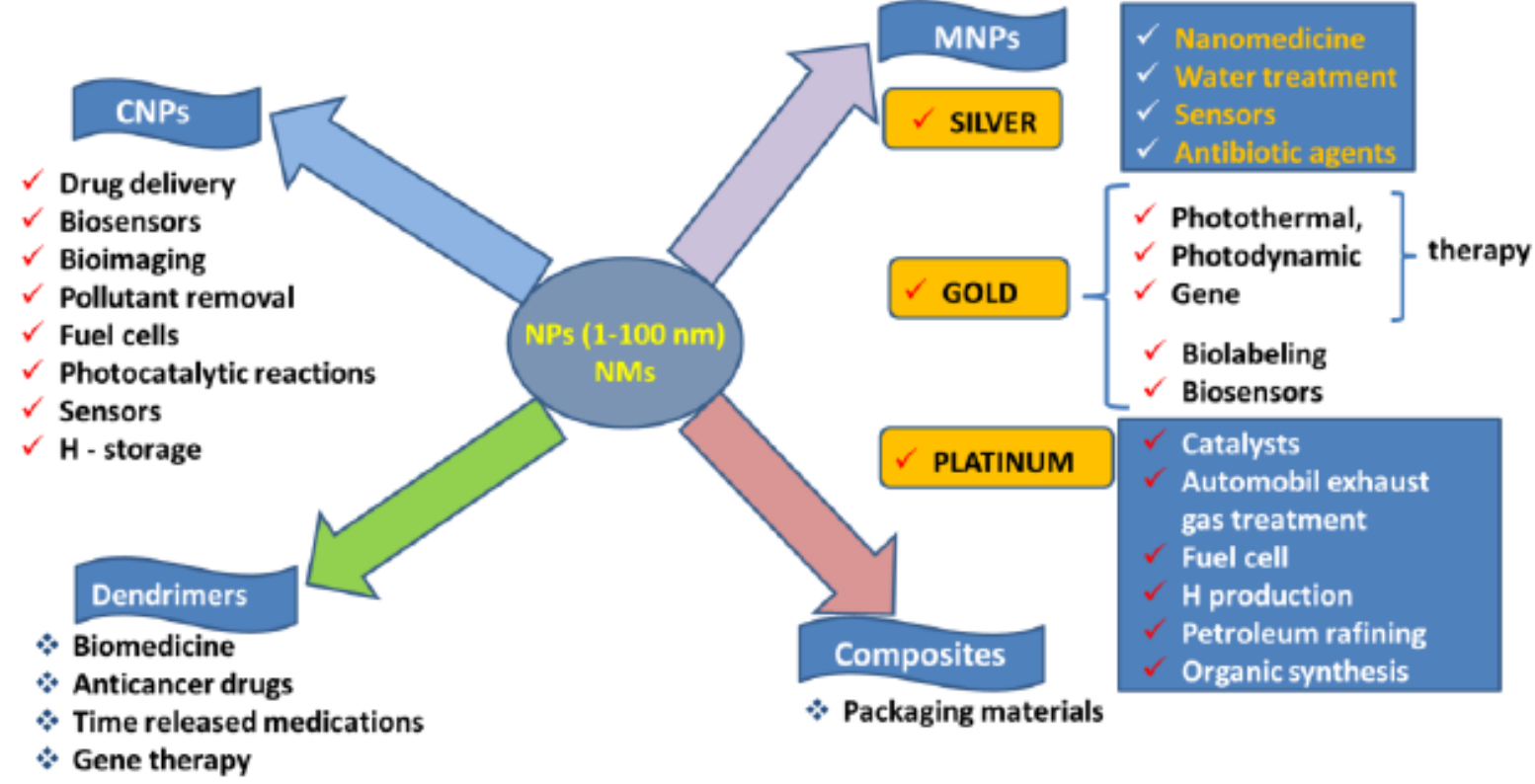

Fig. 11. Classification and wide range of applications of nanomaterials [7]

\section{-Energy}

World-wide energy needs are fossil-based fuels that are provided with the equivalent of approximately 210 million barrels a day. 85 million barrels of this is provided by oil (40\%). Here, the amount of renewable energy is at low levels. Considering the world energy need, it is understood that this situation will gradually increase and the resulting $\mathrm{CO}_{2}$ emission into the atmosphere will rise to alarming levels.

Pyroelectric $\mathrm{ZnO}$ nanowires can convert time-dependent temperature differences into electricity by spontaneous polarization in the conversion based on temperature difference. The said energy conversion takes place with the semiconductor and pyroelectric properties of $\mathrm{ZnO}$. The nanogenerator developed here will be able to meet the energy requirements of nano devices by converting balanced and waste energy. 
Nanotechnologies have great potential for cleaner, more efficient and environmentally friendly energy generation. Energy-related technologies where nanotechnologies can play important roles are as follows: Lighting, Heating, Transport, Renewable energy, Energy storage, Fuel cells, Hydrogen production and storage. Nanofluids are formed by mixing KNTs added into water. With the heat transfer of nanofluidics, $10 \%$ higher efficiency can be obtained in central heating systems.

Depending on the catalysts in fuels, it is possible to use more efficient energy with nanotechnologies. More efficient batteries and accumulators can be developed with lighter and more robust materials. A significant increase in efficiency and effectiveness in the transportation industry can be achieved with such materials. Here, especially lightness and durability are important for more efficient energy use.

Nanotechnologies are used in lithium-ion batteries, which are emphasized for hybrid and electric vehicles, and significant improvements have been made in efficiency and effectiveness (Figure 12).

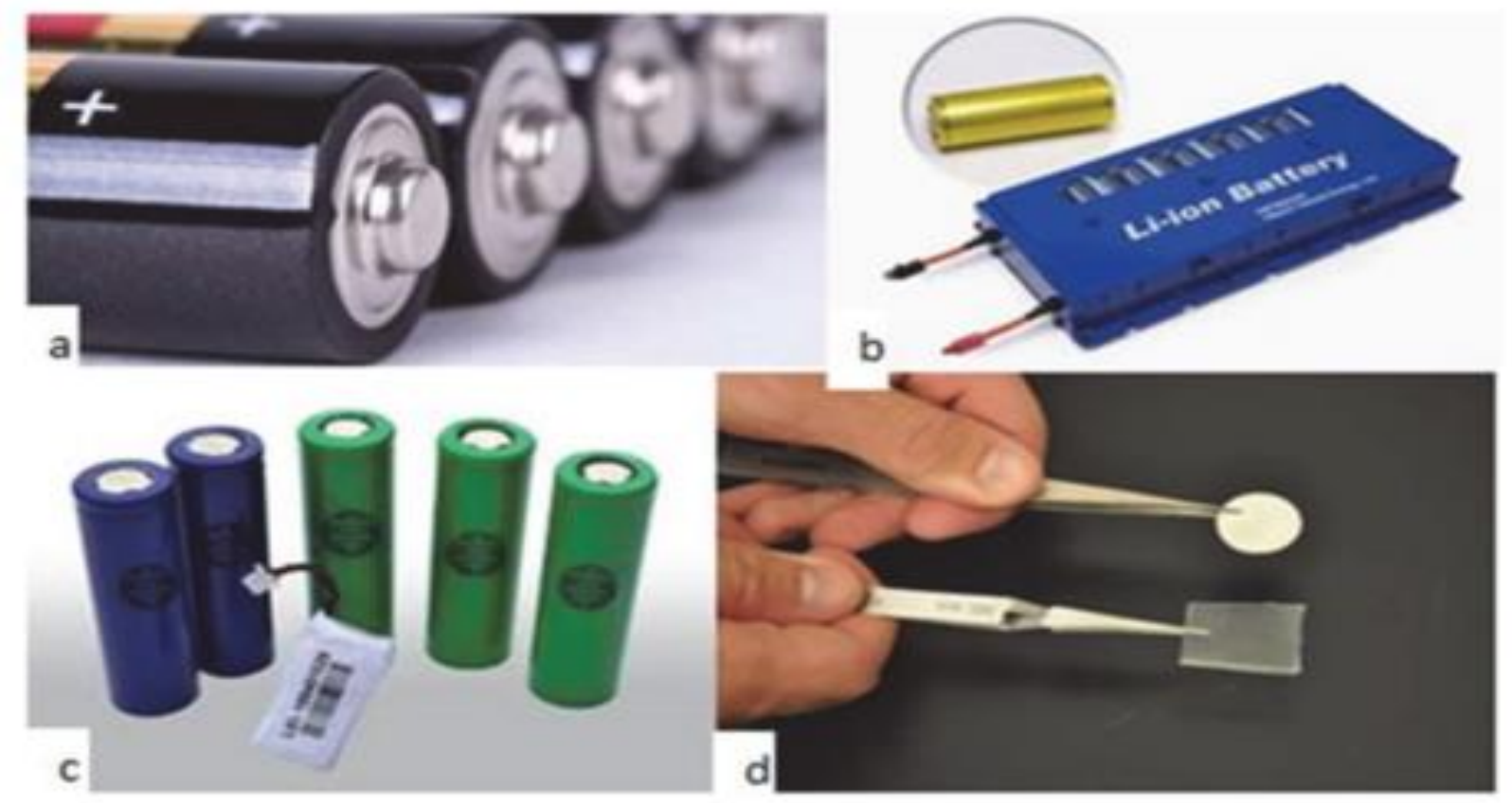

Figure 12. The lithium-ion batteries

\section{-Defense}

Nanotechnological developments have led to the emergence of textile products that have important functions in textile science and technologies. For defense technologies, war uniforms with the following characteristics and qualities, with nanotechnological developments that can meet the requirements expected from uniforms and combat clothing, clothes, camouflage nets, camouflage covers have been developed.

UAVs (Unmanned Aerial Vehicles), which are widely used today for both observation-reconnaissance and attack and destruction purposes, are based on units and special units, which are further reduced and flying, marching and sea flying at micron level for instant short-range and short-field exploration and observation. different mini tools are being developed for.

It is seen that many innovations in nanoscience and nanotechnologies have started to be applied in land, air, sea and space vehicles, which are the most strategic areas in defense fields. Defense tools are becoming areas where nanotechnologies are used in every aspect as advanced technology platforms all over the world in an extremely diverse and wide range. In the demonstration given in the figure on the other page, defense tools in which different nanotechnologies are applied and especially stand out with their "stealth" features are shown in Figure 13. 


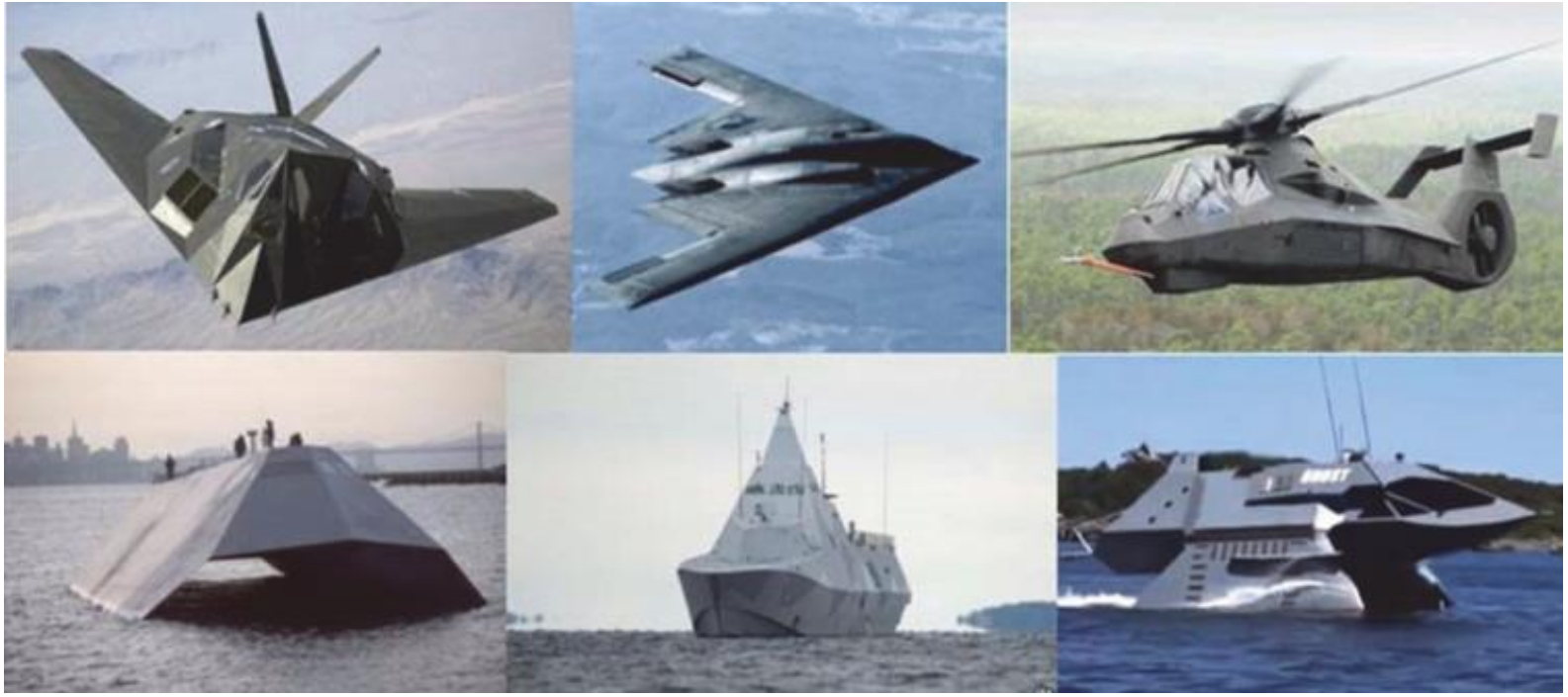

Figure 13. The defense tools in which different nanotechnologies are applied and especially stand out with their "stealth" features [1]

\section{-Environment}

Nanosensor applications that can continuously measure and give warnings and alarms in case of deviation from the given threshold values are becoming widespread in order to monitor air, water and environment pollution. Nanosensors play an indispensable role in monitoring the ecological quality of air, water and environment with precise and precise data. Various nanostructured materials have been developed for the detection of different components.

Potable, clean water is one of the most important needs of almost every society and is generally limited. Especially a large population of the earth has great concerns with the ever-increasing need for water and it gradually becomes one of the most important problems of this century. It has been determined that approximately 780 million people have significant difficulties in accessing potable and clean water resources. It is of great importance to develop innovative, inexpensive, practical and widely used techniques and methods together with nanotechnologies against the limited possibilities of known water treatment and processing, and the production of potable water from sea water [3].

Environmental cleaning (remediation) consists of works that are free from chemical and radiological contaminants and do not endanger human health, together with separation and separation and other processes. Faster and cost-effective cleaning-purification is possible with nanomaterial applications. The most appropriate approach in nanotechnological methods is selectivity and cleaning and elimination of organic / inorganic pollutants (Figure 14).

The use of nSDDs in groundwater cleaning is among the most emphasized nanotechnological issues. It is known that SDDs in granular form have been used for a long time. With nSDDs, it is aimed to create a layer by injecting water to the carrier soil for attachment. In addition, mobile nSDD can be used to create light reactive iron particles and destroy organic pollutants. Successful application of these methods has been demonstrated and a decrease in TCE (trichlorethylene) concentration of $>80 \%$ has been noted in soil injected with $400 \mathrm{~kg}$ of nSDD. 


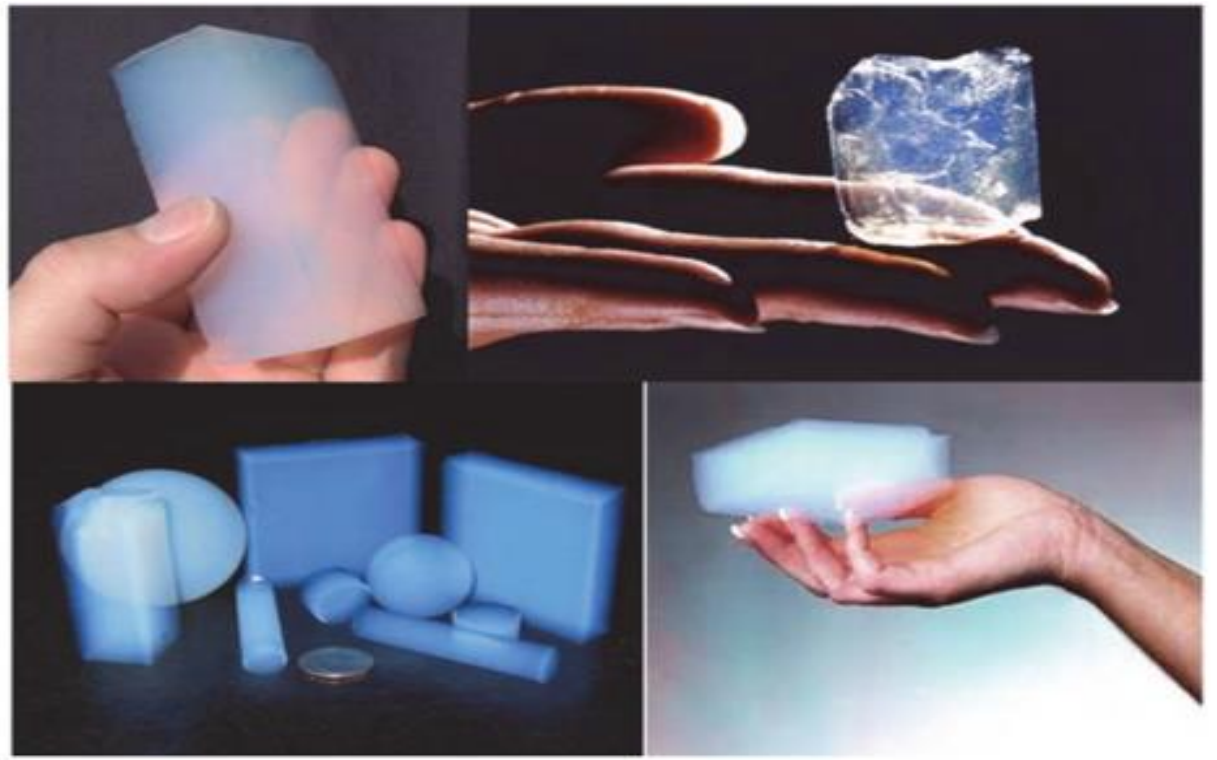

Figure 14. Examples of aerogels that can effectively clean water, sea-ocean, soil against oil spills, various aerogels and silica aerogels samples [1]

-Food and agriculture

Nanotechnology applications in the food industry are handled by the most important companies in this sector. Giant companies such as Nestle, Kraft, Heinz, Unilever support many research and innovation projects and programs in order to lead the future nanofood market (Figure 15). With the development of high nutritional value and delicious products based on "on demand", desired products can be produced with thousands of nanocapsules to taste, colors and additives and vitamins in their content [8].

Starting from the cultivation of food in the soil, production, processing and packaging stages with nanotechnology techniques and devices is called "Nanogood Technology" [9, 10].
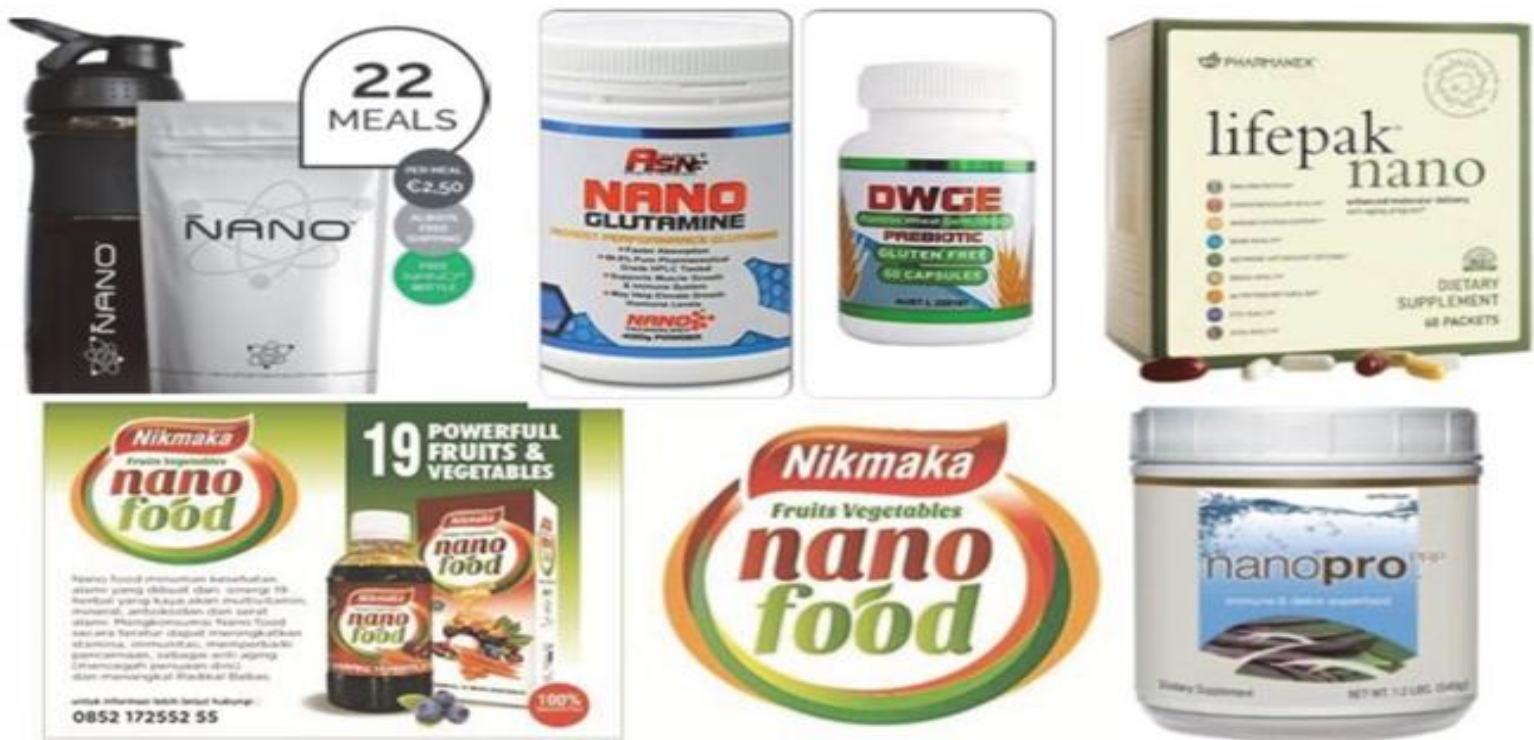

Figure 15. Examples of various nutritious, vitamin and similar food products offered to the world markets

\section{-Other}

It is used in sunscreen content with titanium dioxide, zinc oxide, particle sizes of $20 \mathrm{~nm}$. They are extremely effective in UV filtering. In addition, it has no side effects. In addition to these, fullerens, nanotubes, liposomes, quantum dots are also used by different cosmetic companies in cosmetic products. 
Various improvements and developments in the sports equipment, equipment and tools used in almost every branch of sports can make a difference in relentless competition (Figure 16). More and more high-tech products are being used in the sports industry, which has grown rapidly in recent years and has attracted great attention in all layers of the society. Applications of nanoscience and nanotechnology in this field drew attention in the first pioneering products along with cosmetics.

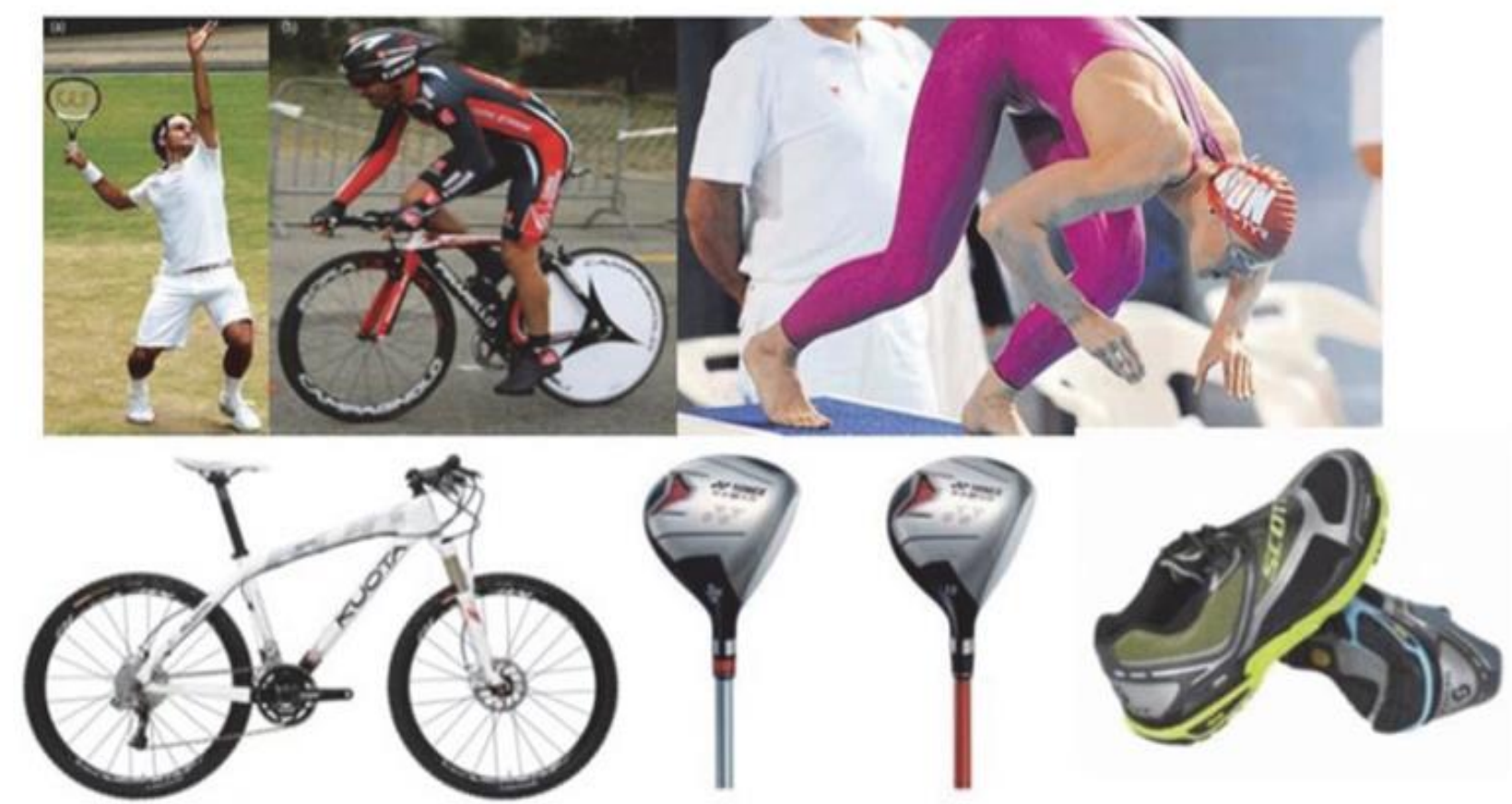

Figure 16. Usage examples of nanotechnologies in various sports and sports equipment

The use of nanotechnologies and nanostructured materials is increasing in construction and architecture. Innovative products are constantly being marketed in the building materials industry, which are more durable, durable, extremely lightweight and have high insulating properties. In addition, new building materials and techniques are also seen in coatings with different properties. In addition to reinforced concrete, glass, wood, brick and metal surface coatings are widely used in coating.

As a matter of fact, "nanotextiles" are widely used in nano-socks (anti-bacterial), dirt-proof, iron-free, wrinklefree pants, curtains, carpets and many textile products, which are among the most common nano products of today. new products are constantly being released.

When we look at it to draw a historical perspective, in fact, we come across examples of "nanotechnological" applications within thousands of years of technological accumulation. In this regard, another discussion centers around the issue that "nanotechnological" scientific and technological approaches are not so new.

\section{Conclusion}

Nanomaterials describe materials in which a single unit of small size (at least one size) is between 1 and 100 $\mathrm{nm}$ (the usual definition of the nanoscale). Materials with a nanoscale structure often have unique optical, electronic, thermo-physical or mechanical properties. The following conclusions can be drawn from the present study:

a. The nanomaterials have extremely small size which having at least one dimension $100 \mathrm{~nm}$ or less. Nanomaterials can be Nanoscale in one dimension, two dimensions, or three dimensions. They can exist in single, fused, aggregated or agglomerated forms with spherical, tubular, and irregular shapes.

b. Carbon-based materials such as carbon nanotubes or fullerenes Inorganic nanoparticles: metal oxides (such as zinc oxide, iron oxide, titanium dioxide and cerium oxide), metals (gold, silver and iron) and 
quantum dots "quantum dots" (cadmium sulfide and cadmium selenide). Gas and liquid phase processes and Photolithography techniques can be used in the production of nanomaterials.

c. Microscopy techniques give images of structures or details too small to be seen with the human eye. In order to be able to examine and investigate any object, TEM, AFM and STM techniques can be used in the imaging of nanomaterials.

d. Carbon-carbon bonds are the strongest in nature. Therefore, the carbon nanotube structure is a "perfect" arrangement of these strong bonds. Carbon nanotubes stand out with their «inert» property, extremely high surface area, high tensile strength, low density, high thermal conductivity and flexible electronic behavior. They can also have high electron conductivity.

e. In composite materials metals, ceramics or polymers can be used as matrix materials. Nanocomposites are composite materials that have two or more components and at least one of the components is the size of the nanometer scale. Nanocomposites are used in a variety of places: For example, water vapor permeability is significantly reduced with nanocomposite coatings. In addition, hard and highly scratch resistant coatings are possible on some inorganic-organic composites, especially glasses and lenses.

f. It is possible to see the nanomaterials in nature including lotus leaves and gecko feet. Therefore, always bright, clean and smooth textiles and building outsides can be build inspiring from lotus leaves as well as to move rapidly in all conditions vertically and inverted on any surface since exciting from gecko feet.

g. Nanomaterials can be used in automobile industry, energy, defense, environment, food and agriculture as well as other sectors such as cosmetics and sports industry.

\section{References}

[1] T. Baykara, Towards the World of Nanotechnologies: Nobel, Ankara, 2016.

[2] F. Trotta and A. Mele (Ed.), "Nanomaterials: Classification and Properties", in Nanosponges: Synthesis and Applications: First Edition, Wiley, London, 2019

[3] P. Sharma and M. Bhargava. "Applications and Characteristics of Nanomaterials in Industrial Environment", International Journal of Civil, Structural, Environmental and Infrastructure Engineering Research and Development, vol. 3, no. 4, October, pp. 63-72, 2013.

[4] S.C. Smith and D.F. Rodrigues, "Carbon-Based nanomaterials for removal of chemical and biological contaminants from water: A review of mechanisms and applications," Carbon, vol. 91, pp. 122-143, 2015.

[5] A. Alagarasi, "Introduction to nanomaterials," in B. Viswanathan (ed.), Nanomaterials, Narosa Publishing House, 2009.

[6] R. Asmatulu, "Nanotechnology Safety in the Automotive Industry", Nanotechnology Safety, New York, Elsevier, pp. 57-72, 2013.

[7] A. Bratovcic. "Different Applications of Nanomaterials and Their Impact on the Environment", SSRG International Journal of Material Science and Engineering (IJMSE), vol. 5, no. 1, pp. 1-7, Jan - April 2019.

[8] M. Shafiq, S. Anjum, C. Hano, I. Anjum and B. H. Abbasi. "An Overview of the Applications of Nanomaterials and Nanodevices in the Food Industry", Foods, vol. 9, no. 148; pp. 1-27, 2020.

[9] S. Belluco, F. Gallocchio, C. Losasso and A. Ricci, "State of art of nanotechnology applications in the meat chain: A qualitative synthesis", Crit. Rev. Food Sci. Nutr, vol. 58, pp. 1084-1096, 2018.

[10] M.R. Mozafari, C. Johnson, S. Hatziantoniou and C. Demetzos, "Nanoliposomes and their applications in food nanotechnology”, J. Liposome Res, vol. 18, pp. 309-327, 2008. 